\title{
Mixed dynamic and static risk-minimization with an application to survivor swaps
}

\author{
Mikkel Dahl \\ Nordea Markets \\ Strandgade 3 \\ DK-0900 Copenhagen $C$ \\ Denmark \\ mikkel.dahl@nordea.com
}

\author{
Sverkel Glar \\ PFA Pension \\ Sundkrogsgade 4 \\ DK-2100 Copenhagen Ø \\ Denmark \\ svg@pfa.dk
}

\author{
Thomas Møller \\ PFA Pension \\ Sundkrogsgade 4 \\ DK-2100 Copenhagen Ø \\ Denmark \\ thm@pfa.dk
}

\begin{abstract}
In the traditional setup, the financial market consists of liquid and dynamically traded financial assets. Here, we extend this setup to include an illiquid asset, which may be traded at fixed, discrete times only. Within this setting of mixed dynamic and static hedging, we adopt the criterion of risk-minimization and minimize the so-called risk process at the fixed trading times for the illiquid asset. The optimal mixed dynamic and static risk-minimizing strategies are compared with the optimal dynamic strategies, and certain correction terms that arise, when trading is restricted to discrete time for the illiquid asset, are identified. We apply the technique for a life insurance company whose liabilities are described by a general insurance payment process. Here, the traditional financial market contains a savings account and a zero coupon bond, which may be traded continuously, and an illiquid mortality derivative, traded at fixed times. We provide numerical illustrations with survivor swaps and compare the minimum obtainable risk with the risk for the optimal dynamic strategies.
\end{abstract}

Key words: Risk-minimization, hedging, life insurance, risk management, stochastic mortality, longevity, mortality derivative, survivor swap.

JEL Classification: G10.

Mathematics Subject Classification (2000): 62P05, 91B28.

This version: August 25, 2009 


\section{Introduction}

In this paper we study the situation, where the financial market consists of a number of liquid assets and an illiquid asset, and derive risk-minimizing hedging strategies for a general payment stream. The criterion of risk-minimization was originally proposed by Föllmer and Sondermann (1986) as an approach to hedging in incomplete markets. By minimizing a certain squared error process, they derived optimal hedging strategies for a contingent claim with a fixed finite time horizon. This idea was extended to payment processes in Møller (2001), where insurance payment processes driven by Markov chains were studied. For a review of risk-minimization and related criteria, see Schweizer (2001). Here, we derive risk-minimizing hedging strategies within a setting of mixed dynamic and static hedging by requiring that the risk process is minimized at the fixed trading points for the illiquid asset. In this way, the dynamic investments in the traditional risky assets and the static investments in the illiquid asset are determined uniquely. The optimal deposit in the savings account is then determined by subsequently minimizing the risk process between the trading times for the illiquid asset. The optimal mixed dynamic and static risk-minimizing strategies are related to the fully dynamic strategies for the case where the illiquid asset may also be traded dynamically. We point out similarities and identify correction terms that arise when trading is restricted to discrete times for the illiquid asset.

Schweizer (1994) studied the problem of hedging under restricted information. This framework encompasses the situation where the hedger only observes the prices of the risky assets at fixed discrete time points. In that setting, Schweizer (1994) derived risk-minimizing strategies. The main results state that risk-minimizing strategy under restricted information may be obtained as a suitable projection of the risk-minimizing strategies under normal/full information. For the special case of piecewise constant information, the resulting optimal strategies are typically not piecewise constant, since they typically include some deterministic time-dependence between the time points where new information is available. For comparison, we include a short review of these results.

As a main application of our general results we consider a life insurance company which is exposed to both interest rate risk and mortality/longevity risk. Here there exists a liquid market for bonds and interest rate swaps which can be used to manage the interest rate risk, whereas mortality and longevity risk can be controlled by reinsurance or by investing in the illiquid market for mortality derivatives. Main examples of mortality derivatives are longevity bonds and survivor swaps, see e.g. Cairns, Blake and Dowd (2008) for a recent review of mortality derivatives and mortality risk management. A longevity bond is an annuity bond, whose coupon payments are linked to the development of a given underlying portfolio of individuals. Typically, the coupons would be proportional to the number of survivors in this portfolio. The market value of the longevity bond depends on the current interest rate level and on the expected future number of survivors. If the mortality decreases, both the market value of the longevity bond and the market value associated with a portfolio of life annuities would increase. Thus, by investing in longevity bonds, the insurance company would in principle be able to hedge the risk associated with general changes in the underlying mortality. With a survivor swap, the insurance company would receive the difference between the current number of survivors and the expected number of survivors. We study the model presented in Dahl, Melchior and Møller (2008), where survivor swaps were used for dynamic hedging together with more traditional assets. Here, we examine the consequences of restricting trading of the survivor 
swap to a set of fixed times. As a special case, we analyse the situation where the survivor swap is purchased at time 0 and held until maturity of the contract.

The paper is organized as follows. Section 2 presents the general model, and Section 3 contains a brief review on risk-minimization for payment processes and the main results when hedging under restricted information. In Section 4, the general mixed dynamic and static risk-minimizing strategies are derived. The mortality model and survivor swaps are introduced in Section 5. Section 6 contains the optimal mixed dynamic and static strategies for the survivor swap, and Section 7 presents a numerical study of the efficiency of the mixed dynamic and static strategies.

\section{General framework}

Consider a filtered probability space $(\Omega, \mathcal{F}, P)$ with a filtration $\mathbb{F}$ describing the amount of information available. We study a financial market with a savings account $B$ and $d+1$ risky assets with discounted price process $(X, Y)=\left(X^{1}, \ldots, X^{d}, Y\right)$. Here, $X$ is interpreted as the price process associated with traditional liquid and dynamically traded financial assets, whereas $Y$ is the price process associated with an additional illiquid asset, which may be traded at fixed discrete times only. We fix throughout a martingale measure $Q$ such that the $\mathbb{F}$-adapted process $(X, Y)$ is a $(d+1)$-dimensional martingale.

We consider a payment process $A$ which describes the liabilities of the hedger. More precisely, $A(t)$ is the accumulated outflow of payments during $[0, t]$ from the hedger. In addition, we introduce the discounted payment process $A^{*}(t)$ given by $A^{*}(t)=A(0)+$

$\int_{0}^{t} B(u)^{-1} d A(u)$. The process $A$ is assumed to be adapted to the filtration $\mathbb{F}$.

\section{Risk-minimization for payment processes}

In this section, we consider the usual situation where both $X$ and $Y$ can be traded dynamically and briefly review classical results on risk-minimization in continuous time within this setting, see Föllmer and Sondermann (1986) and Møller (2001).

A strategy is a sufficiently integrable process $\varphi=(\xi, \vartheta, \eta)$, where $\xi$ is a $d$-dimensional $\mathbb{F}$ predictable process, $\vartheta$ is $\mathbb{F}$-predictable and $\eta$ is $\mathbb{F}$-adapted. The process $\xi$ is the number of liquid risky financial assets held, $\vartheta$ is the number of illiquid risky assets, and $\eta$ is the discounted deposit in the savings account. The discounted value at time $t$ associated with the strategy is given by $V^{*}(t, \varphi)=\xi(t) X(t)+\vartheta(t) Y(t)+\eta(t)$. We restrict ourselves to so-called 0 -admissible strategies, i.e. strategies with terminal value 0 , that is $V^{*}(T, \varphi)=0$. The cost process at time $t$ is defined by

$$
C(t, \varphi)=V^{*}(t, \varphi)-\int_{0}^{t} \xi(u) d X(u)-\int_{0}^{t} \vartheta(u) d Y(u)+A^{*}(t) .
$$

According to this definition, the accumulated costs at time $t$ are simply the discounted value $V^{*}(t, \varphi)$ of the investment portfolio, reduced by discounted trading gains and added discounted payments. We emphasize that the strategies considered are not necessarily self-financing.

A strategy $\varphi$ is called risk-minimizing if it minimizes the risk process $R(\cdot, \varphi)$ defined by

$$
R(t, \varphi)=\mathrm{E}^{Q}\left[(C(T, \varphi)-C(t, \varphi))^{2} \mid \mathcal{F}(t)\right]
$$


for all $t$. It follows from Föllmer and Sondermann (1986) and Møller (2001) that the strategy in this case can be determined from the Galtchouk-Kunita-Watanabe decomposition given by

$$
\begin{aligned}
V^{*, Q}(t) & =\mathrm{E}^{Q}\left[A^{*}(T) \mid \mathcal{F}(t)\right] \\
& =V^{*, Q}(0)+\int_{0}^{t} \xi^{A}(u) d X(u)+\int_{0}^{t} \vartheta^{A}(u) d Y(u)+L^{A}(t),
\end{aligned}
$$

where $\xi^{A}$ and $\vartheta^{A}$ are predictable processes, and where $L^{A}$ is a zero-mean $Q$-martingale which is orthogonal to $(X, Y)$. More precisely, there exists a unique 0-admissible riskminimizing strategy $\varphi^{*}=\left(\xi^{*}, \vartheta^{*}, \eta^{*}\right)$, given by

$$
\left(\xi^{*}(t), \vartheta^{*}(t), \eta^{*}(t)\right)=\left(\xi^{A}(t), \vartheta^{A}(t), V^{*, Q}(t)-\xi^{A}(t) X(t)-\vartheta^{A}(t) Y(t)-A^{*}(t)\right),
$$

see Møller (2001, Theorem 2.1). The minimum obtainable risk process is given by

$$
R\left(t, \varphi^{*}\right)=\mathrm{E}^{Q}\left[\left(L^{A}(T)-L^{A}(t)\right)^{2} \mid \mathcal{F}(t)\right] .
$$

\subsection{Hedging under restricted information}

Schweizer (1994) studies the situation where the strategy $\varphi=(\xi, \vartheta, \eta)$ is adapted to two smaller filtrations $\mathbb{G} \subseteq \mathbb{G}^{\prime} \subseteq \mathbb{F}$. More precisely, it is assumed that the process $(\xi, \vartheta)$ is $\mathbb{G}$-predictable and $\eta$ is $\mathbb{G}^{\prime}$-adapted. One example where this could be of interest is the case where the prices for the risky assets are only available at discrete times and where hedging can only be based on this restricted information.

We review here the main results in the simplest situation where there is only one risky asset $X$. We assume that $d\langle X\rangle(t)=\sigma(t) d t$, i.e. the variance process of $X$ is absolutely continuous with respect to the Lebesgue measure. In this case, Schweizer (1994, Theorem 2.5) proves the existence of a unique $\left(G_{r}, \mathbb{G}^{\prime}\right)$-risk-minimizing strategy for a payment $H$ at the fixed time $T$ corresponding to a payment process $A^{*}(t)=H 1_{\{t \geq T\}}$. More precisely, this strategy is given by

$$
\begin{aligned}
& \xi^{*, G, G^{\prime}}(t)=\frac{\mathrm{E}^{Q}\left[\sigma(t) \xi^{A}(t) \mid \mathcal{G}(t-)\right]}{\mathrm{E}^{Q}[\sigma(t) \mid \mathcal{G}(t-)]}, \\
& \eta^{*, G_{i}, G^{\prime}}(t)=\mathrm{E}^{Q}\left[H-\xi^{*, G_{i}, G_{t}^{\prime}}(t) X(t) \mid \mathcal{G}^{\prime}(t)\right] .
\end{aligned}
$$

In particular, if $\mathbb{G}$ is piecewise continuous, i.e. $\mathcal{G}(t)=\mathcal{G}\left(t_{k}\right)$ for $t \in\left[t_{k}, t_{k+1}\right), 0=t_{0}<t_{1}<$ $\cdots<t_{n}=T$, we get for $t \in\left(t_{k}, t_{k+1}\right]$

$$
\xi^{*, G, G_{t}^{\prime}}(t)=\frac{\mathrm{E}^{Q}\left[\sigma(t) \xi^{A}(t) \mid \mathcal{G}\left(t_{k}\right)\right]}{\mathrm{E}^{Q}\left[\sigma(t) \mid \mathcal{G}\left(t_{k}\right)\right]} .
$$

We note that this strategy is not in general piecewise continuous. In particular, this implies that trading will in general take place continuously and between the times $t_{k}$ where information about the price process is being updated.

\section{Mixed dynamic and static hedging}

In this section, we derive a mixed continuous and discrete time strategy for market $\left(B^{*}, X, Y\right)$, where $B^{*}=1$ is the discounted savings account, $X$ are the liquid risky fi- 
nancial assets and $Y$ is the illiquid financial asset. We assume that $Y$ can be traded in discrete time only.

We study risk-minimizing hedging strategies in the case, where the illiquid asset may be traded at fixed (deterministic) times $0=t_{0}<t_{1}<\cdots<t_{n}=T$ only. We assume that both $X$ and $Y$ are $\mathbb{F}$-adapted, such that the both price processes may be observed at any time $t$ and not only the discrete time points $t_{i}, i=0, \ldots, n$. In this setting, we modify the definition of a trading strategy slightly. Here, a trading strategy is a process $\varphi=(\xi, \vartheta, \eta)$ where the process $(\xi, \vartheta)$ is $\mathbb{F}$-predictable, $\vartheta$ is piecewise constant on the intervals $\left(t_{i}, t_{i+1}\right], i=0, \ldots, n-1$, and $\eta$ is adapted. In addition, the strategy satisfies certain integrability conditions. We focus on 0 -admissible strategies, i.e. $V^{*}(T, \varphi)=0$. Thus, we assume that $Y$ is observed at any time, whereas it may only be traded at the fixed times $0=t_{0}<t_{1}<\cdots<t_{n}=T$, i.e. during the period $\left(t_{i}, t_{i+1}\right]$ we keep $\vartheta(t)$ fixed and equal to $\vartheta\left(t_{i}\right)$, which is $\mathcal{F}\left(t_{i}\right)$-measurable. Note that with this definition of $\vartheta$, the process is predictable, as required.

We work with the following optimality criterion:

Definition 4.1 A strategy $\varphi^{*}=\left(\xi^{*}, \vartheta^{*}, \eta^{*}\right)$ with $\vartheta^{*}$ piecewise constant on the intervals $\left(t_{i}, t_{i+1}\right], i=0, \ldots, n-1$, is said to be mixed discrete- and continuous-time risk-minimizing if $\varphi^{*}=\left(\xi^{*}, \vartheta^{*}, \eta^{*}\right)$ minimizes the risk process $R\left(t_{i}, \varphi\right)=R\left(t_{i}, \xi, \vartheta, \eta\right)$ for $i=0, \ldots, n-1$, and, in addition, $(\xi, \eta)$ minimizes $R\left(t, \xi, \vartheta^{*}, \eta\right)$ for all $t$ among all strategies with $\vartheta=\vartheta^{*}$.

The first part of the definition states that the strategy should minimize the risk process at the discrete trading points. This condition is in fact sufficient to fix the optimal values of $(\xi, \vartheta)$, whereas $\eta$ is not determined uniquely at all times $t \in[0, T]$ from this condition. The second part of the definition states that given the optimal choice of $\vartheta$, the pair $(\xi, \eta)$ should in addition minimize the risk process at all remaining time points. This additional condition is indeed sufficient to fix the choice of $\eta$ uniquely.

\subsection{The Galtchouk-Kunita-Watanabe decomposition}

As in the traditional case, the continuous-time Galtchouk-Kunita-Watanabe decomposition (3.3) of the intrinsic value process under $Q$ is useful for determining the riskminimizing strategy. Recall that $L^{A}$ is orthogonal to $(X, Y)$, whereas the two martingales $X$ and $Y$ will typically not be orthogonal. We therefore decompose $Y$ as

$$
d Y(t)=\xi^{Y}(t) d X(t)+d L^{Y}(t)
$$

using the Galtchouk-Kunita-Watanabe decomposition for the martingale $Y$. Thus, $L^{Y}$ is a zero-mean $Q$-martingale, which is orthogonal to $X$ and $\xi^{Y}$ is $\mathbb{F}$-predictable. In (4.1), the dynamics of the discounted price process $Y$ are decomposed into a term which is related to the price process $X$ and a term which is orthogonal to $X$. This decomposition will be useful later, where we assume that $Y$ can be traded in discrete time only. In this case the decomposition quantifies the risk associated with $Y$ which can be hedged by investing in $X$.

If we insert (4.1) in the continuous time Galtchouk-Kunita-Watanabe decomposition (3.3), we obtain

$$
V^{*, Q}(t)=V^{*, Q}(0)+\int_{0}^{t}\left(\xi^{A}(u)+\vartheta^{A}(u) \xi^{Y}(u)\right) d X(u)+\int_{0}^{t} \vartheta^{A}(u) d L^{Y}(u)+L^{A}(t) .
$$


Thus, we have rewritten the decomposition (3.3) into an alternative decomposition with orthogonal processes $X$ and $L^{Y}$. Note that the process $\vartheta^{A}$ now also appears in the integral with respect to $X$.

As mentioned above, we assume that $Y$ can be traded at the fixed times $0=t_{0}<t_{1}<$ $\cdots<t_{n}=T$ only, i.e., for $t \in\left(t_{i}, t_{i+1}\right]$ we have $\vartheta(t)=\vartheta\left(t_{i}\right)$. This means that we are looking for a decomposition with an integrand $\widehat{\vartheta}^{A}(t)$, which is piecewise constant on the intervals $\left(t_{i}, t_{i+1}\right]$. We therefore rewrite the decomposition (4.2) on the form

$$
\begin{aligned}
V^{*, Q}\left(t_{i}\right)= & V^{*, Q}\left(t_{0}\right)+\int_{0}^{t_{i}}\left(\xi^{A}(u)+\vartheta^{A}(u) \xi^{Y}(u)\right) d X(u) \\
& +\sum_{j=1}^{i}\left(\int_{t_{j-1}}^{t_{j}} \vartheta^{A}(u) d L^{Y}(u)+\Delta L^{A}\left(t_{j}\right)\right),
\end{aligned}
$$

where $\Delta L^{A}\left(t_{j}\right)=L^{A}\left(t_{j}\right)-L^{A}\left(t_{j-1}\right)$.

\subsection{The risk-minimizing strategy}

For any given strategy $\varphi=(\xi, \vartheta, \eta)$, where $\vartheta$ is not necessarily piecewise constant, we can use decomposition (4.1) to rewrite the cost process (3.1) as

$$
C(t, \varphi)=V^{*}(t, \varphi)-\int_{0}^{t}\left(\xi(u)+\vartheta(u) \xi^{Y}(u)\right) d X(u)-\int_{0}^{t} \vartheta(u) d L^{Y}(u)+A^{*}(t) .
$$

Since $\varphi$ is 0 -admissible, we get

$$
C(T, \varphi)=-\int_{0}^{T}\left(\xi(u)+\vartheta(u) \xi^{Y}(u)\right) d X(u)-\int_{0}^{T} \vartheta(u) d L^{Y}(u)+A^{*}(T) .
$$

Using the decomposition (4.2) for $t=T$, and since $V^{*, Q}(T)=A^{*}(T)$, we get

$$
\begin{aligned}
A^{*}(T)=V^{*, Q}(T) & =V^{*, Q}(t)+\int_{t}^{T}\left(\xi^{A}(u)+\vartheta^{A}(u) \xi^{Y}(u)\right) d X(u) \\
& +\int_{t}^{T} \vartheta^{A}(u) d L^{Y}(u)+\left(L^{A}(T)-L^{A}(t)\right) .
\end{aligned}
$$

In order to minimize the risk process $(3.2)$, we need to study the future $\operatorname{costs} C(T, \varphi)-$ $C(t, \varphi)$. Using (4.4)-(4.6), we obtain the following expression for the future costs

$$
\begin{aligned}
C(T, \varphi)-C(t, \varphi)= & \left(V^{*, Q}(t)-A^{*}(t)-V^{*}(t, \varphi)\right) \\
& +\int_{t}^{T}\left(\xi^{A}(u)+\vartheta^{A}(u) \xi^{Y}(u)-\left(\xi(u)+\vartheta(u) \xi^{Y}(u)\right)\right) d X(u) \\
& +\int_{t}^{T}\left(\vartheta^{A}(u)-\vartheta(u)\right) d L^{Y}(u)+\left(L^{A}(T)-L^{A}(t)\right) .
\end{aligned}
$$

Since the martingales $X, L^{Y}$ and $L^{A}$ are orthogonal, we get

$$
\begin{aligned}
R(t, \varphi) & =\mathrm{E}^{Q}\left[(C(T, \varphi)-C(t, \varphi))^{2} \mid \mathcal{F}(t)\right] \\
& =\left(V^{*, Q}(t)-A^{*}(t)-V^{*}(t, \varphi)\right)^{2} \\
& +\mathrm{E}^{Q}\left[\left(\int_{t}^{T}\left(\xi^{A}(u)+\vartheta^{A}(u) \xi^{Y}(u)-\left(\xi(u)+\vartheta(u) \xi^{Y}(u)\right)\right) d X(u)\right)^{2} \mid \mathcal{F}(t)\right] \\
& +\mathrm{E}^{Q}\left[\left(\int_{t}^{T}\left(\vartheta^{A}(u)-\vartheta(u)\right) d L^{Y}(u)\right)^{2} \mid \mathcal{F}(t)\right]+\mathrm{E}^{Q}\left[\left(L^{A}(T)-L^{A}(t)\right)^{2} \mid \mathcal{F}(t)\right] .
\end{aligned}
$$


If $(\xi, \vartheta, \eta)$ could be chosen freely, we could eliminate the first three terms by taking $\xi=\xi^{A}$, $\vartheta=\vartheta^{A}$, and by choosing $\eta$ such that $V^{*}(\varphi)=V^{*, Q}-A^{*}$. This would give the usual riskminimizing strategy (3.4) for the case, where all assets are traded continuously.

Here, part of the strategy $\vartheta$ is required to be piecewise constant, such that we cannot in general eliminate the squared term involving $\int\left(\vartheta^{A}-\vartheta\right) d L^{Y}$, and we cannot minimize the risk process at any time $t$. For any given $\vartheta$ we can, however, eliminate the first two terms by taking $\xi=\xi^{A}+\xi^{Y}\left(\vartheta^{A}-\vartheta\right)$ and ensuring that $V(\varphi)=V^{*, Q}-A^{*}$ by choosing $\eta$ appropriately.

Now consider the risk process $R\left(t_{j}, \varphi\right)$ at the discrete time points $t_{j}, j=0, \ldots, n-1$, and a strategy $\varphi$ where $\vartheta$ is piecewise continuous. It follows that we need to minimize the terms

$$
\begin{aligned}
& \mathrm{E}^{Q}\left[\left(\int_{t_{j}}^{T}\left(\vartheta^{A}(u)-\vartheta(u)\right) d L^{Y}(u)\right)^{2} \mid \mathcal{F}\left(t_{j}\right)\right] \\
& \left.=\sum_{i=j}^{n-1} \mathrm{E}^{Q}\left[\left(\int_{t_{i}}^{t_{i+1}} \vartheta^{A}(u) d L^{Y}(u)-\vartheta\left(t_{i}\right) \Delta L^{Y}\left(t_{i+1}\right)\right)\right)^{2} \mid \mathcal{F}\left(t_{j}\right)\right],
\end{aligned}
$$

where we have used that $\vartheta$ is piecewise constant on the intervals $\left(t_{i}, t_{i+1}\right]$ and introduced $\Delta L^{Y}\left(t_{i+1}\right)=L^{Y}\left(t_{i+1}\right)-L^{Y}\left(t_{i}\right)$. In addition, we have exploited that $Y$ is a martingale. Thus, we need to minimize the terms

$$
\left.\mathrm{E}^{Q}\left[\left(\int_{t_{i}}^{t_{i+1}} \vartheta^{A}(u) d L^{Y}(u)-\vartheta\left(t_{i}\right) \Delta L^{Y}\left(t_{i+1}\right)\right)\right)^{2} \mid \mathcal{F}\left(t_{i}\right)\right],
$$

where $\vartheta\left(t_{i}\right)$ is $\mathcal{F}\left(t_{i}\right)$-measurable, and where we have used the tower property to obtain a conditional expected value with respect to $\mathcal{F}\left(t_{i}\right)$. Differentiating in (4.10) with respect to $\vartheta\left(t_{i}\right)$, we get the solution

$$
\widehat{\vartheta}\left(t_{i}\right)=\frac{\mathrm{E}^{Q}\left[\int_{t_{i}}^{t_{i+1}} \vartheta^{A}(u) d L^{Y}(u) \Delta L^{Y}\left(t_{i+1}\right) \mid \mathcal{F}\left(t_{i}\right)\right]}{\mathrm{E}^{Q}\left[\left(\Delta L^{Y}\left(t_{i+1}\right)\right)^{2} \mid \mathcal{F}\left(t_{i}\right)\right]}
$$

for $i=0, \ldots, n-1$, which minimizes (4.9) and hence minimizes the risk process (4.8) at times $t_{0}, \ldots, t_{n-1}$. Thus, we have shown the following.

Theorem 4.2 The unique mixed discrete- and continuous-time risk-minimizing strategy associated with the discounted payment process $A^{*}$ is given by

$$
\begin{aligned}
\widehat{\vartheta}^{*}(t) & =\widehat{\vartheta}^{A}\left(t_{j-1}\right)=\frac{E^{Q}\left[\int_{t_{j-1}}^{t_{j}} \vartheta^{A}(u) d L^{Y}(u) \Delta L^{Y}\left(t_{j}\right) \mid \mathcal{F}\left(t_{j-1}\right)\right]}{E^{Q}\left[\left(\Delta L^{Y}\left(t_{j}\right)\right)^{2} \mid \mathcal{F}\left(t_{j-1}\right)\right]}, \quad t \in\left(t_{j-1}, t_{j}\right], \\
\widehat{\xi}^{*}(t) & =\widehat{\xi}^{A}(t)=\xi^{A}(t)+\xi^{Y}(t)\left(\vartheta^{A}(t)-\widehat{\vartheta}^{A}\left(t_{j-1}\right)\right), \quad t \in\left(t_{j-1}, t_{j}\right], \\
\eta(t) & =V^{*, Q}(t)-A^{*}(t)-\widehat{\xi}^{A}(t) X(t)-\widehat{\vartheta}^{A}\left(t_{j-1}\right) Y(t), \quad t \in\left(t_{j-1}, t_{j}\right] .
\end{aligned}
$$

We note that discrete-time trading in the asset $Y$ also affects the investment in $X$. More precisely, the investment strategy for $X$ is given by the original strategy $\xi^{A}$ from continuous time and a correction term $\xi^{Y}\left(\vartheta^{A}-\widehat{\vartheta}^{A}\right)$, which is the strategy for $Y$ in continuous time reduced by the $Y$-strategy from discrete time and multiplied by $\xi^{Y}$. 


\subsection{A mixed continuous- and discrete-time decomposition}

The risk-minimizing strategy can be found from a modified version of the GaltchoukKunita-Watanabe decomposition. We rewrite (4.3) in order to obtain the mixed continuousand discrete-time version of the Galtchouk-Kunita-Watanabe decomposition. This is done by adding and subtracting the approximation $\widehat{\vartheta}^{A}\left(t_{j-1}\right) \Delta L^{Y}\left(t_{j}\right)$. It follows that

$$
\begin{aligned}
V^{*, Q}\left(t_{i}\right)= & V^{*, Q}\left(t_{0}\right)+\int_{0}^{t_{i}}\left(\xi^{A}(u)+\vartheta^{A}(u) \xi^{Y}(u)\right) d X(u) \\
& +\sum_{j=1}^{i}\left(\widehat{\vartheta}^{A}\left(t_{j-1}\right) \Delta L^{Y}\left(t_{j}\right)+\Delta \widehat{L}^{A}\left(t_{j}\right)\right),
\end{aligned}
$$

where

$$
\Delta \widehat{L}^{A}\left(t_{j}\right)=\int_{t_{j-1}}^{t_{j}} \vartheta^{A}(u) d L^{Y}(u)-\widehat{\vartheta}^{A}\left(t_{j-1}\right) \Delta L^{Y}\left(t_{j}\right)+\Delta L^{A}\left(t_{j}\right)
$$

and where we have used the quantity $\widehat{\vartheta}^{A}\left(t_{j-1}\right)$ defined in Lemma 4.2 above. Finally, we use that

$$
\Delta L^{Y}\left(t_{j}\right)=\Delta Y\left(t_{j}\right)-\int_{t_{j-1}}^{t_{j}} \xi^{Y}(u) d X(u),
$$

to obtain a decomposition which involves $X$ and $Y$. We collect the results in the following proposition.

Proposition 4.3 Consider the market $\left(B^{*}, X, Y\right)$ and the continuous-time GaltchoukKunita-Watanabe decomposition (3.3) for the intrinsic value process. Assume that $Y$ can be traded in discrete time only. The corresponding mixed continuous- and discrete-time version of the Galtchouk-Kunita-Watanabe decomposition is given by

$$
\begin{aligned}
V^{*, Q}\left(t_{i}\right)= & V^{*, Q}\left(t_{0}\right)+\int_{0}^{t_{i}} \widehat{\xi}^{A}(u) d X(u) \\
& +\sum_{j=1}^{i}\left(\widehat{\vartheta}^{A}\left(t_{j-1}\right) \Delta Y\left(t_{j}\right)+\Delta \widehat{L}^{A}\left(t_{j}\right)\right),
\end{aligned}
$$

for $i=1, \ldots, n$, where

$$
\begin{aligned}
\widehat{\vartheta}^{A}\left(t_{j-1}\right) & =\frac{E^{Q}\left[\int_{t_{j-1}}^{t_{j}} \vartheta^{A}(u) d L^{Y}(u) \Delta L^{Y}\left(t_{j}\right) \mid \mathcal{F}\left(t_{j-1}\right)\right]}{E^{Q}\left[\left(\Delta L^{Y}\left(t_{j}\right)\right)^{2} \mid \mathcal{F}\left(t_{j-1}\right)\right]}, \\
\widehat{\xi}^{A}(t) & =\xi^{A}(t)+\xi^{Y}(t)\left(\vartheta^{A}(t)-\widehat{\vartheta}^{A}\left(t_{j-1}\right)\right), \quad t \in\left[t_{j-1}, t_{j}\right),
\end{aligned}
$$

and

$$
\Delta \widehat{L}^{A}\left(t_{j}\right)=\int_{t_{j-1}}^{t_{j}} \vartheta^{A}(u) d L^{Y}(u)-\widehat{\vartheta}^{A}\left(t_{j-1}\right) \Delta L^{Y}\left(t_{j}\right)+\Delta L^{A}\left(t_{j}\right) .
$$


Note that since we can trade the illiquid asset $Y$ in discrete time only, the integral in (3.3) with respect to $Y$ is replaced in (4.13) by a sum of the terms $\widehat{\vartheta}^{A}\left(t_{j-1}\right) \Delta Y\left(t_{j}\right)$.

Proof of Proposition 4.3: We have to show the orthogonality in (4.12). Clearly, $\widehat{L}^{A}$ and $X$ are orthogonal, since both $L^{A}$ and $L^{Y}$ are orthogonal to $X$. It therefore only remains to show that $Y$ and $\widehat{L}^{A}$ are orthogonal. From (3.3) we have that $Y$ is orthogonal to $L^{A}$. To show the orthogonality between $Y$ and the first two terms in $\widehat{L}^{A}$, we use decomposition (4.1). Since $X$ is orthogonal to both $\widehat{L}^{A}$ and $L^{A}$ it holds that $\int \xi^{Y} d X$ is obviously orthogonal to the first two terms in $\widehat{L}^{A}$. Furthermore, we have that

$$
\begin{aligned}
& \mathrm{E}^{Q}\left[\Delta L^{Y}\left(t_{j}\right)\left(\int_{t_{j-1}}^{t_{j}} \vartheta^{A}(u) d L^{Y}(u)-\widehat{\vartheta}^{A}\left(t_{j-1}\right) \Delta L^{Y}\left(t_{j}\right)\right) \mid \mathcal{F}\left(t_{j-1}\right)\right] \\
&= \mathrm{E}^{Q}\left[\Delta L ^ { Y } ( t _ { j } ) \left(\int_{t_{j-1}}^{t_{j}} \vartheta^{A}(u) d L^{Y}(u)\right.\right. \\
&\left.\left.-\frac{\mathrm{E}^{Q}\left[\int_{t_{j-1}}^{t_{j}} \vartheta^{A}(u) d L^{Y}(u) \Delta L^{Y}\left(t_{j}\right) \mid \mathcal{F}\left(t_{j-1}\right)\right]}{\mathrm{E}^{Q}\left[\left(\Delta L^{Y}\left(t_{j}\right)\right)^{2} \mid \mathcal{F}\left(t_{j-1}\right)\right]} \Delta L^{Y}\left(t_{j}\right)\right) \mid \mathcal{F}\left(t_{j-1}\right)\right] \\
&= \mathrm{E}^{Q}\left[\int_{t_{j-1}}^{t_{j}} \vartheta^{A}(u) d L^{Y}(u) \Delta L^{Y}\left(t_{j}\right) \mid \mathcal{F}\left(t_{j-1}\right)\right] \\
&= 0 . \\
& \mathrm{E}^{Q}\left[\int_{t_{j-1}}^{t_{j}} \vartheta^{A}(u) d L^{Y}(u) \Delta L^{Y}\left(t_{j}\right) \mid \mathcal{F}\left(t_{j-1}\right)\right] \mathrm{E}^{Q}\left[\left(\Delta L^{Y}\left(t_{j}\right)\right)^{2} \mid \mathcal{F}\left(t_{j-1}\right)\right] \\
&=
\end{aligned}
$$

That is, the product of $\Delta L^{Y}$ and the first two parts of $\Delta \widehat{L}^{A}$ is a $Q$-martingale and therefore orthogonal. We conclude that the orthogonality requirement in (4.12) is fulfilled.

Remark 4.4 For $t \in\left(t_{i}, t_{i+1}\right)$, the decomposition (4.12) may be written as

$$
\begin{aligned}
V^{*, Q}(t)= & V^{*, Q}\left(t_{i}\right)+\int_{t_{i}}^{t} \widehat{\xi}^{A}(u) d X(u)+\widehat{\vartheta}^{A}\left(t_{i}\right)\left(Y(t)-Y\left(t_{i}\right)\right) \\
& +\int_{t_{i}}^{t} \vartheta^{A}(u) d L^{Y}(u)-\widehat{\vartheta}^{A}\left(t_{i}\right)\left(L^{Y}(t)-L^{Y}\left(t_{i}\right)\right)+\left(L^{A}(t)-L^{A}\left(t_{i}\right)\right) .
\end{aligned}
$$

Here, the second line represents the orthogonal terms.

\section{$5 \quad$ A stochastic mortality model and survivor swaps}

In this section, we describe the model introduced in Dahl et al. (2008) in order to examine the mixed dynamic and static risk-minimizing strategies. 


\subsection{The basic model}

The filtration $\mathbb{F}$ is generated by a standard Brownian motion $W^{r, Q}$ driving the interest rate, a 2-dimensional standard Brownian motion $W^{\mu, Q}$ driving the mortality intensity, and a 2-dimensional counting process $N$ which counts the number of deaths in two portfolios. The three processes $W^{r, Q}, W^{\mu, Q}$ and $N$ are associated with the sub-filtrations $\mathbb{G}$, II and $\mathbb{H}$, respectively. We assume that $W^{r, Q}$ and $\left(W^{\mu, Q}, N\right)$ are independent.

\subsection{The standard financial market}

The basic financial market consists of two traded assets; a savings account with price process $B$ (a risk free asset), and a zero coupon bond with price process $P(\cdot, T)$, where $T$ is the time of maturity. We assume that both assets can be traded freely in continuous time. The short rate is modeled by a Vasiček model and has the following dynamics under $Q$

$$
d r(t)=\left(\gamma^{r}-\delta^{r} r(t)\right) d t+\sigma^{r} d W^{r, Q}(t),
$$

where $W^{r, Q}$ is a standard Brownian motion under $Q$. A Vasiček model has the desirable property of an affine term structure, which means that the zero coupon bond price processes can be written on the form

$$
P(t, T)=\mathrm{E}^{Q}\left[e^{-\int_{t}^{T} r(u) d u} \mid \mathcal{F}_{t}\right]=e^{A^{r}(t, T)-B^{r}(t, T) r(t)} .
$$

Here, $A^{r}$ and $B^{r}$ are given by

$$
\begin{aligned}
& B^{r}(t, T)=\frac{1}{\delta^{r}}\left(1-e^{-\delta^{r}(T-t)}\right) \\
& A^{r}(t, T)=\frac{\left(B^{r}(t, T)-T+t\right)\left(\gamma^{r} \delta^{r}-\frac{1}{2}\left(\sigma^{r}\right)^{2}\right)}{\left(\delta^{r}\right)^{2}}-\frac{\left(\sigma^{r} B^{r}(t, T)\right)^{2}}{4 \delta^{r}}
\end{aligned}
$$

see e.g. Björk (2004). By applying Itô's formula and using that the discounted price process is a $Q$-martingale, we get the bond price dynamics under $Q$

$$
d P(t, T)=r(t) P(t, T) d t-\sigma^{r} B^{r}(t, T) P(t, T) d W^{r, Q}(t) .
$$

The dynamics of the savings account are

$$
d B(t)=r(t) B(t) d t, \quad B(0)=1 .
$$

\subsection{The insurance portfolios}

We consider two different portfolios; one representing an insurance portfolio and the other representing some population. The mortality intensities for the two portfolios are

$$
\mu_{j}(x, t)=\mu_{j}^{0}(x+t) \zeta_{j}(x, t), \quad j=1,2,
$$

where $\mu_{j}^{0}(x+t)$ is the initial mortality intensity at age $x+t$, and the mortality improvement process $\zeta=\left(\zeta_{1}, \zeta_{2}\right)$ is defined by the $Q$-dynamics

$$
d \zeta_{j}(x, t)=\left(\gamma_{j}(x, t)-\delta_{j}(x, t) \zeta_{j}(x, t)\right) d t+\sqrt{\zeta_{j}(x, t)} \sigma_{j}(x, t) d W^{\mu, Q}(t) .
$$


Here $j=1,2$ is referring to the insurance portfolio and the population, respectively, $\sigma_{j}$ is a two-dimensional vector controlling the possible correlation between the processes, and $W^{\mu, Q}$ is a two-dimensional standard Brownian motion.

Using Itô's lemma, we get the following $Q$-dynamics for the mortality intensities

$$
d \mu_{j}(x, t)=\left(\gamma_{j}^{\mu}(x, t)-\delta_{j}^{\mu}(x, t) \mu_{j}(x, t)\right) d t+\sqrt{\mu_{j}(x, t)} \sigma_{j}^{\mu}(x, t) d W^{\mu, Q}(t), \quad j=1,2,
$$

where

$$
\begin{aligned}
\gamma_{j}^{\mu}(x, t) & =\mu_{j}^{0}(x+t) \gamma_{j}(x, t), \\
\delta_{j}^{\mu}(x, t) & =\delta_{j}(x, t)-\frac{\frac{d}{d t} \mu_{j}^{0}(x+t)}{\mu_{j}^{0}(x+t)} \\
\sigma_{j}^{\mu}(x, t) & =\sqrt{\mu_{j}^{0}(x+t)} \sigma_{j}(x, t) .
\end{aligned}
$$

The assumption needed to ensure strict positivity on the mortality intensity is given by

$$
2 \gamma_{j}^{\mu}(x, t) \geq\left(\sigma_{j}^{\mu}(x, t)\right)\left(\sigma_{j}^{\mu}(x, t)\right)^{\operatorname{tr}}=\left(\sigma_{j, 1}^{\mu}(x, t)\right)^{2}+\left(\sigma_{j, 2}^{\mu}(x, t)\right)^{2},
$$

where $a^{\text {tr }}$ denotes the vector $a$ transposed.

Let $n_{j}, j=1,2$, be the number of lives in each portfolio, all aged $x$ years at time 0 . We assume that the portfolios consist of different lives, and that the lifetimes in each portfolio are mutually independent and identically distributed conditional on the mortality intensities.

We define the survival probabilities under $Q$ by

$$
\mathcal{S}_{j}^{Q}(x, t, T)=\mathrm{E}^{Q}\left[e^{-\int_{t}^{T} \mu_{j}^{Q}(x, u) d u} \mid \mathcal{F}(t)\right] .
$$

Since we have an affine mortality structure, see e.g. Dahl (2004), the $Q$-survival probabilities $\mathcal{S}_{j}^{Q}(x, t, T), j=1,2$, are given by

$$
\mathcal{S}_{j}^{Q}(x, t, T)=e^{A_{j}^{\mu, Q}(x, t, T)-B_{j}^{\mu, Q}(x, t, T) \mu_{j}^{Q}(x, t)},
$$

where $A_{j}^{\mu, Q}$ and $B_{j}^{\mu, Q}$ are determined from a set of differential equations, see Dahl and Møller (2006).

The forward mortality intensities under $Q$ are for $j=1,2$ given by

$$
f^{\mu_{j}, Q}(x, t, T)=-\frac{\partial}{\partial T} \log \mathcal{S}_{j}^{Q}(x, t, T)=\mu_{j}^{Q}(x, t) \frac{\partial}{\partial T} B_{j}^{\mu, Q}(x, t, T)-\frac{\partial}{\partial T} A_{j}^{\mu, Q}(x, t, T) .
$$

The remaining lifetimes at time 0 are random variables defined as $T_{j, 1}, \ldots, T_{j, n_{j}}$, and the $Q$-probability of one individual surviving time $t$ given the development of the mortality intensity until time $t$, is given by

$$
Q\left(T_{j, 1}>t \mid \mathcal{I}(t)\right)=e^{-\int_{0}^{t} \mu_{j}(x, s) d s}, \quad j=1,2 .
$$

Furthermore, we describe the number of deaths in portfolio $j$ at time $t \in[0, T]$ by the counting process $N_{j}(x)=\left(N_{j}(x, t)\right)_{t \in[0, T]}$, that is

$$
N_{j}(x, t)=\sum_{i=1}^{n_{j}} 1_{\left\{T_{j, i} \leq t\right\}}, \quad j=1,2 .
$$


The stochastic intensity process $\lambda_{j}(x)=\left(\lambda_{j}(x, t)\right)_{t \in[0, T]}$ related to $N_{j}(x)$ is given by

$$
\lambda_{j}(x, t) d t=\mathrm{E}^{Q}\left[d N_{j}(x, t) \mid \mathcal{H}(t-) \vee \mathcal{I}(t)\right]=\left(n_{j}-N_{j}(x, t-)\right) \mu_{j}(x, t) d t .
$$

Finally, we let the $Q$-martingales $M_{j}^{Q}(x)=\left(M_{j}^{Q}(x, t)\right)_{t \in[0, T]}$ be defined by the dynamics

$$
d M_{j}^{Q}(x, t)=d N_{j}(x, t)-\lambda_{j}(x, t) d t, \quad j=1,2 .
$$

\subsection{Numerical illustrations of the mortality model}

The initial mortality intensity is assumed to follow the Gompertz-Makeham model

$$
\mu_{j}^{0}(x+t)=a_{j}+b_{j} c_{j}^{x+t}
$$

and the parameters for the initial mortality intensity used in the numerical examples throughout are listed in Table 1 . The parameters associated with the insurance portfolio are taken from Dahl and Møller (2006), and the modified list of parameters for the population is taken from Dahl et al. (2008). The parameters for the mortality develop-

\begin{tabular}{cccc}
\hline \hline Portfolio $(j)$ & $a$ & $b$ & $c$ \\
\hline 1 & 0.0001340 & 0.0000353 & 1.1020000 \\
2 & 0.0001360 & 0.0000350 & 1.1030000 \\
\hline \hline
\end{tabular}

Table 1: Gompertz-Makeham parameters.

ment processes are given in Table 2. We note that $\sigma_{2,1}=0$. That is, $W_{1}^{\mu, Q}$ affects the uncertainty in the insurance portfolio only, and $W_{2}^{\mu, Q}$ is used to model the uncertainty of the mortality intensities in general. The parametrization of the development process in

\begin{tabular}{ccccc}
\hline \hline Portfolio $(j)$ & $\gamma_{j}(x, t)$ & $\delta_{j}(x, t)$ & $\sigma_{j, 1}(x, t)$ & $\sigma_{j, 2}(x, t)$ \\
\hline 1 & 0.0001800 & 0.0080 & 0.006 & 0.018 \\
2 & 0.0001805 & 0.0081 & 0.000 & 0.019 \\
\hline \hline
\end{tabular}

Table 2: Parameters for the development process.

Table 2 leads to an expected relative yearly decline of $0.80 \%$ in the insurance portfolio and $0.81 \%$ in the population (the $\delta_{j}$ value). The mean reversion level is given by $\frac{\gamma_{j}}{\delta_{j}}$; see Dahl and Møller (2006). That is, the mean reversion level is 0.0225 for the insurance portfolio and 0.0223 for the population, which are both negligible given the time horizon used here since we take $\zeta_{1}(x, 0)=\zeta_{2}(x, 0)=1$.

In Figure 1 the density functions of $\zeta_{j}$ are plotted for different time horizons. The first main observation from Figure 1 is that due to the decreasing trend, the average values of $\zeta_{j}$ are decreasing. The second major observation is that the variability of $\zeta_{j}$, and thus the systematic mortality risk, is an increasing function of the time horizon. That is, when we look at long time horizons the systematic risk is more important compared to short time horizons. We note that $\zeta_{1}$ has a slightly higher expected value compared to $\zeta_{2}$. 

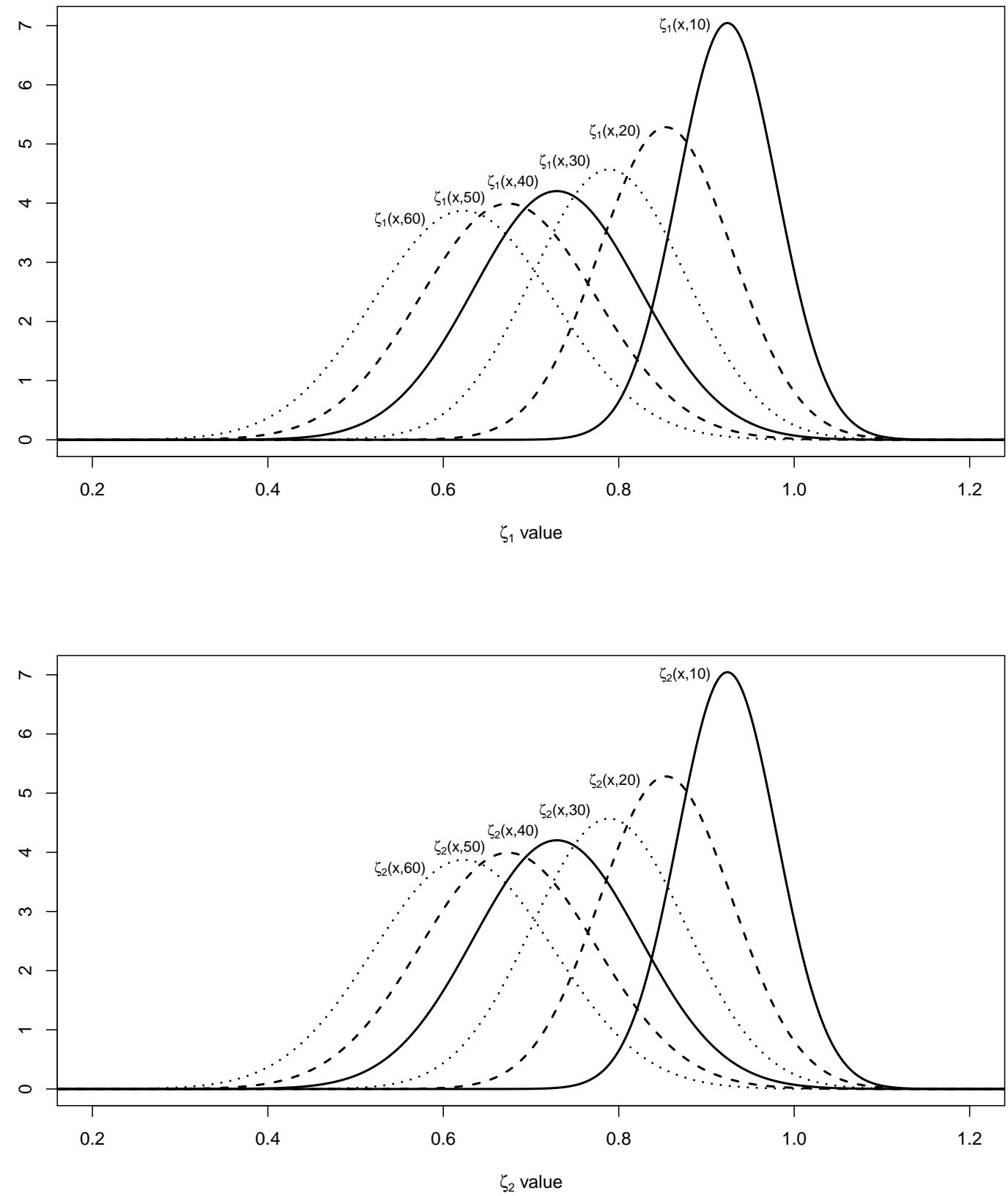

Figure 1: Density of $\zeta_{j}$ for different time horizons (the densities are based on 50,000 simulations with 30 steps per year). 
$\zeta_{1}$

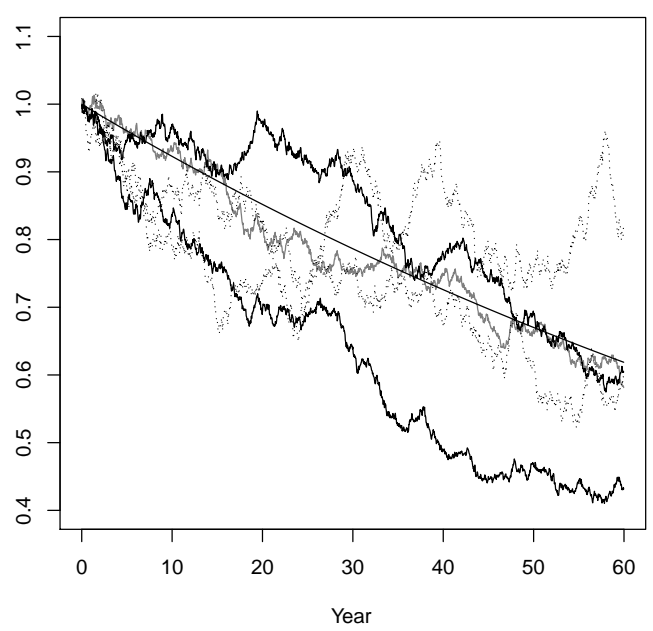

$\zeta_{2}$

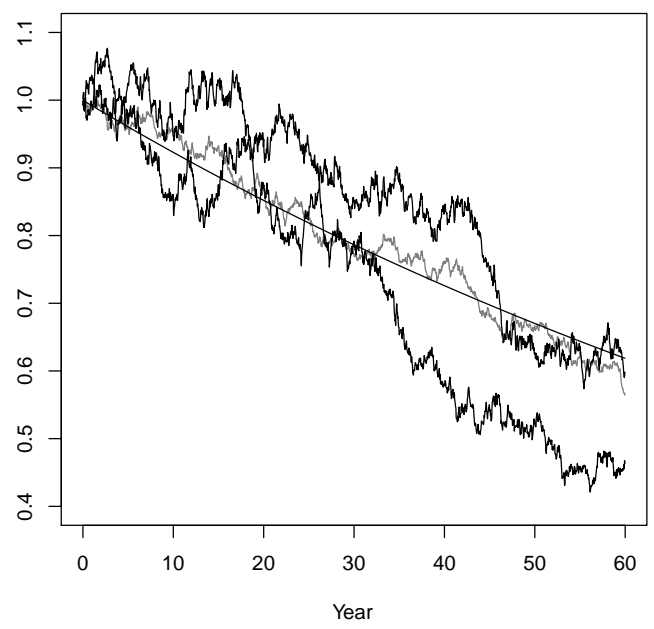

Figure 2: Realizations of the development process $\zeta_{j}$ in different scenarios. The grey curve is a realization with the parameters from Table 2 , and the black line is the associated trend. For $\zeta_{1}$, the solid black curves are realizations with $\sigma_{1,1}$ doubled, and the dotted curves are realizations with $\sigma_{1,2}$ doubled. For $\zeta_{2}$, the solid black curves are realizations with $\sigma_{2,2}$ doubled.

In order to illustrate the fluctuation when we change $\sigma$, we have included some realizations of $\zeta_{j}$ in Figure 2 for different values of $\sigma$; we double $\sigma_{1,1}$ and $\sigma_{1,2}$ (one at a time) in the insurance portfolio case, and we double $\sigma_{2,2}$ on the population.

In Table 3 we list the quantiles of $\zeta(x, 20)$. We note that the quantiles for $\zeta_{1}$ are slightly higher than those for $\zeta_{2}$ due to a lower expected relative yearly decline. Furthermore, we note that doubling $\sigma_{1,2}$ has a greater impact on the uncertainty than doubling $\sigma_{1,1}$. This is in line with the intentions, since we want $W_{1}^{\mu, Q}$ to play a minor role on the mortality intensity compared to $W_{2}^{\mu, Q}$.

\begin{tabular}{cccccccccc}
\hline \hline Portfolio $(j)$ & $\sigma_{j, 1}$ & $\sigma_{j, 2}$ & $5 \%$ & $10 \%$ & $25 \%$ & $50 \%$ & $75 \%$ & $90 \%$ & $95 \%$ \\
\hline 1 & 0.006 & 0.018 & 0.734 & 0.760 & 0.804 & 0.854 & 0.906 & 0.954 & 0.985 \\
1 & 0.012 & 0.018 & 0.719 & 0.748 & 0.798 & 0.855 & 0.914 & 0.969 & 1.003 \\
1 & 0.006 & 0.036 & 0.637 & 0.683 & 0.765 & 0.859 & 0.960 & 1.055 & 1.114 \\
2 & 0.000 & 0.019 & 0.733 & 0.758 & 0.802 & 0.853 & 0.904 & 0.953 & 0.982 \\
2 & 0.000 & 0.038 & 0.627 & 0.676 & 0.759 & 0.859 & 0.964 & 1.062 & 1.124 \\
\hline \hline
\end{tabular}

Table 3: Quantiles of $\zeta(x, 20)$ for various parameter values (the numbers are based on 50,000 simulations with 30 steps per year). 


\subsection{The insurance contract}

We study the general life insurance contract from Dahl et al. (2008), which allows for a single premium at time 0 , a continuous premium paid until retirement contingent on survival, a single payment at retirement, a single payment upon death before retirement and a life annuity payment. The payment process associated with the insurance portfolio is defined by

$$
\begin{aligned}
d A(t)= & -n_{1} \pi^{s}(0) d 1_{\{t \geq 0\}}-\pi^{c}(t)\left(n_{1}-N_{1}(x, t)\right) 1_{\{0 \leq t<\bar{T}\}} d t \\
& +\left(n_{1}-N_{1}(x, \bar{T})\right) a^{r}(\bar{T}) d 1_{\{t \geq \bar{T}\}}+a^{p}(t)\left(n_{1}-N_{1}(x, t)\right) 1_{\{\bar{T} \leq t \leq T\}} d t \\
& +a^{d}(t) 1_{\{0 \leq t<\bar{T}\}} d N_{1}(x, t),
\end{aligned}
$$

where $n_{1}$ is the number of lives in the insurance portfolio, $N_{1}(x, t)$ is the number of deaths during $[0, t]$ in the portfolio, $\bar{T}$ is the time of retirement and $T$ is the end of the insurance period. The single premium at time 0 is denoted $\pi^{s}$, the continuously paid premium is denoted $\pi^{c}$ and the benefits are labeled $a^{r}, a^{p}$ and $a^{d}$ representing the retirement payment, the annuity payment and the payment upon death before retirement, respectively. It is assumed that $\pi^{c}, a^{p}$ and $a^{d}$ are piecewise continuous function.

The intrinsic value process of the discounted payment process $A^{*}$ is defined by the following conditional expected value

$$
\begin{aligned}
V^{*, Q}(t) & =\mathrm{E}^{Q}\left[\int_{0-}^{T} d A^{*}(\tau) \mid \mathcal{F}(t)\right]=\mathrm{E}^{Q}\left[\int_{0-}^{T} e^{-\int_{0}^{\tau} r(u) d u} d A(\tau) \mid \mathcal{F}(t)\right] \\
& =\int_{0-}^{t} e^{-\int_{0}^{\tau} r(u) d u} d A(\tau)+\mathrm{E}^{Q}\left[\int_{t}^{T} e^{-\int_{0}^{\tau} r(u) d u} d A(\tau) \mid \mathcal{F}(t)\right] \\
& =A^{*}(t)+\widetilde{V}^{*, Q}(t)=A^{*}(t)+\left(n_{1}-N_{1}(x, t)\right) \widetilde{V}_{p}^{*, Q}(t), \quad 0 \leq t \leq T,
\end{aligned}
$$

where $\widetilde{V}^{*, Q}$ is called the discounted market reserve and represents the discounted conditional expected value of future payments. In addition, we have introduced $\widetilde{V}_{p}^{*, Q}(t)$, which

is interpreted as the individual market reserve. An explicit expression for $\widetilde{V}_{p}^{*, Q}(t)$ can be found in Dahl and Møller (2006).

The next lemma regarding the intrinsic value process of an insurance portfolio is taken from Dahl et al. (2008). It gives a stochastic representation of the insurance contract, which will be very useful later on.

Lemma 5.1 Let $A^{*}$ be the general discounted payment process for the insurance portfolio introduced in (5.4). The intrinsic value process admits the representation

$$
V^{*, Q}(t)=V^{*, Q}(0)+\int_{0}^{t} \nu^{V, Q}(u) d M_{1}^{Q}(x, u)+\int_{0}^{t} \eta^{V, Q}(u) d W^{r, Q}(u)+\int_{0}^{t} \rho^{V, Q}(u) d W^{\mu, Q}(u),
$$

where

$$
\begin{aligned}
\nu^{V, Q}(t)= & B(t)^{-1} a^{d}(t)-\widetilde{V}_{p}^{*, Q}(t) \\
\eta^{V, Q}(t)= & -\sigma^{r}\left(n_{1}-N_{1}(x, t-)\right)\left(\int_{t}^{T} B^{r}(t, u) P^{*}(t, u) S_{1}^{Q}(x, t, u)\right. \\
& \times\left(a^{d}(u) f^{\mu_{1}, Q}(x, t, u)-\pi^{c}(u) 1_{\{0 \leq u \leq \bar{T}\}}+a^{p}(u) 1_{\{\bar{T} \leq u \leq T\}}\right) d u \\
& \left.+B^{r}(t, \bar{T}) P^{*}(t, \bar{T}) S_{1}^{Q}(x, t, \bar{T}) a^{r}(\bar{T}) 1_{\{t<\bar{T}\}}\right)
\end{aligned}
$$


and where

$$
\begin{aligned}
& \rho_{j}^{V, Q}(t) \\
& =-\sigma_{1, j}^{\mu}(x, t) \sqrt{\mu_{1}(x, t)}\left(n_{1}-N_{1}(x, t-)\right)\left(\int_{t}^{T} P^{*}(t, u) B_{1}^{\mu, Q}(x, t, u) S_{1}^{Q}(x, t, u)\right. \\
& \times\left(a^{d}(u)\left(f^{\mu_{1}, Q}(x, t, u)-\frac{\frac{\partial}{\partial u} B_{1}^{\mu, Q}(x, t, u)}{B_{1}^{\mu, Q}(x, t, u)}\right)-\pi^{c}(u) 1_{\{0 \leq u \leq \bar{T}\}}+a^{p}(u) 1_{\{\bar{T} \leq u \leq T\}}\right) d u \\
& \left.+P^{*}(t, \bar{T}) B_{1}^{\mu, Q}(x, t, \bar{T}) S_{1}^{Q}(x, t, \bar{T}) a^{r}(\bar{T}) 1_{\{t<\bar{T}\}}\right), \quad j=1,2 .
\end{aligned}
$$

\subsection{Survivor swaps}

The survivor swap is a financial instrument, where one exchanges a fixed expected number of survivors with the actual number of survivors in an agreed portfolio on a fixed period of time, see e.g. Dowd, Blake, Cairns and Dawson (2006). That is, when the contract is settled, both parties (the buyer and the seller) agree on a fixed survival probability at time 0 given by

$$
{ }_{t} \widetilde{p}_{x}=e^{-\int_{0}^{t} \widetilde{\mu}^{0}(x, u) d u},
$$

where $\widetilde{\mu}^{0}$ is the fixed mortality intensity. The survivor swap is also called a mortality swap in the literature.

We describe the survivor swap by a payment process $A_{j}^{\text {swap }}$, where $j=1,2$ represents the insurance portfolio and the population, respectively. The dynamics of the payment process are given by

$$
d A_{j}^{\mathrm{swap}}(x, t)=\left(n_{j}-N_{j}(x, t)\right) d t-n_{j} \widetilde{p}_{x} d t .
$$

The term $\left(n_{j}-N_{j}(x, t)\right)$ is the number of survivors in portfolio $j$ at time $t$, and we see that there is a payment, if the number of survivors differs from the agreed fixed number of survivors. The contract where the owner receives the fixed payments and pays the variable payments connected with the stochastic number of deaths is called a receiver swap, and if the owner takes the other side, the swap is called a payer swap.

Now, we introduce the conditional expected discounted value of $A_{j}^{\text {swap }}$ given by $Z_{j}^{*, Q}$, where we condition on all information available at time $t$ and take the expected value under some fixed martingale measure $Q$. The process is called the intrinsic value process and is given by

$$
\begin{aligned}
Z_{j}^{*, Q}(x, t) & =\mathrm{E}^{Q}\left[\int_{0}^{T} d A_{j}^{*, \operatorname{swap}}(x, u) \mid \mathcal{F}(t)\right] \\
& =\mathrm{E}^{Q}\left[\int_{0}^{T} e^{-\int_{0}^{u} r(\tau) d \tau} d A_{j}^{\mathrm{swap}}(x, u) \mid \mathcal{F}(t)\right] .
\end{aligned}
$$

By rewriting (5.6), we obtain

$$
\begin{aligned}
Z_{j}^{*, Q}(x, t) & =A_{j}^{*, \operatorname{swap}}(x, t)+e^{-\int_{0}^{t} r(\tau) d \tau} \mathrm{E}^{Q}\left[\int_{t}^{T} e^{-\int_{t}^{u} r(\tau) d \tau} d A_{j}^{\mathrm{swap}}(x, u) \mid \mathcal{F}(t)\right] \\
& =A_{j}^{*, \operatorname{swap}}(x, t)+\widetilde{Z}_{j}^{*, Q}(x, t)
\end{aligned}
$$


where $\widetilde{Z}_{j}^{*, Q}$ is called the discounted market value of future payments. Note that the market value depends on the choice of measure. Exploited the independence between $r$ and $(\mu, N)$ yields the following expression for $\widetilde{Z}_{j}^{*, Q}$

$$
\begin{aligned}
\widetilde{Z}_{j}^{*}(x, t) & =e^{-\int_{0}^{t} r(\tau) d \tau} \mathrm{E}^{Q}\left[\int_{t}^{T} e^{-\int_{t}^{u} r(\tau) d \tau}\left(\left(n_{j}-N_{j}(x, u)\right)-n_{j} u \widetilde{p}_{x}\right) d u \mid \mathcal{F}(t)\right] \\
& =\int_{t}^{T} P^{*}(t, u) \mathrm{E}^{Q}\left[n_{j}-N_{j}(x, u) \mid \mathcal{F}(t)\right] d u-n_{j} \int_{t}^{T} P^{*}(t, u)_{u} \widetilde{p}_{x} d u \\
& =\left(n_{j}-N_{j}(x, t)\right) \int_{t}^{T} P^{*}(t, u) \mathcal{S}_{j}^{Q}(x, t, u) d u-n_{j t} \widetilde{p}_{x} \int_{t}^{T} P^{*}(t, u)_{u-t} \widetilde{p}_{x+t} d u,
\end{aligned}
$$

where $P^{*}(t, u)$ is the discounted price at time $t$ of a zero coupon bond with maturity $u$, which is given by

$$
P^{*}(t, u)=\mathrm{E}^{Q}\left[e^{-\int_{0}^{u} r(\tau) d \tau} \mid \mathcal{F}(t)\right], \quad t \leq u
$$

The following lemma is taken from Dahl et al. (2008).

Lemma 5.2 A survivor swap on portfolio $j$ with fixed survival probability ${ }_{t} \widetilde{p}_{x}$ admits the representation

$$
d Z_{j}^{*, Q}(x, t)=\nu_{j}^{Z, Q}(t) d M_{j}^{Q}(x, t)+\eta_{j}^{Z, Q}(t) d W^{r, Q}(t)+\rho_{j}^{Z, Q}(t) d W^{\mu, Q}(t),
$$

where $\rho_{j}^{Z, Q}=\left(\rho_{j, 1}^{Z, Q}, \rho_{j, 2}^{Z, Q}\right)$ and

$$
\begin{aligned}
\nu_{j}^{Z, Q}(t)= & -\int_{t}^{T} P^{*}(t, u) \mathcal{S}_{j}^{Q}(x, t, u) d u, \\
\eta_{j}^{Z, Q}(t)= & -\sigma^{r}\left(n_{j}-N_{j}(x, t-)\right) \int_{t}^{T} B^{r}(t, u) P^{*}(t, u) \mathcal{S}_{j}^{Q}(x, t, u) d u \\
& +n_{j} \widetilde{p}_{x} \sigma^{r} \int_{t}^{T} B^{r}(t, u) P^{*}(t, u)_{u-t} \widetilde{p}_{x+t} d u, \\
\rho_{j, i}^{Z, Q}(t)= & -\sigma_{j, i}^{\mu}(x, t) \sqrt{\mu_{j}(x, t)}\left(n_{j}-N_{j}(x, t-)\right) \\
& \times \int_{t}^{T} B_{j}^{\mu, Q}(t, u) P^{*}(t, u) \mathcal{S}_{j}^{Q}(x, t, u) d u, \quad i=1,2 .
\end{aligned}
$$

We see that there are three types of risk associated with the value of the survivor swap: the process $M_{j}^{Q}$ drives the unsystematic mortality risk, the interest rate risk is related to $W^{r, Q}$, and $W^{\mu, Q}=\left(W_{1}^{\mu, Q}, W_{2}^{\mu, Q}\right)$ generates the systematic mortality risk.

\section{Risk-minimization with a survivor swap}

We now turn to study more specific cases in discrete time. We derive risk-minimizing strategies in the markets $\left(B, P, Z_{j}\right)$ for $j=1,2$, where $Z_{1}$ is a survivor swap on the insurance portfolio and $Z_{2}$ is a survivor swap on the population. We consider two different discrete time setups; one where the swap can be traded at time 0 only, and a second where the swap can be traded at time 0 and time 30 only. 


\subsection{Parameters for numerical study}

We apply the parameters from Dahl et al. (2008). Thus, we consider an insurance portfolio with policy-holders aged 30 at time 0 , who pay a continuous premium of $\pi^{c}(t)=0.2$ during $[0, \bar{T}]$, where $\bar{T}=30$. In case of a death at time $t$ the insurance company pays a lump sum of $a^{d}(t)=5 \cdot 1_{\{0 \leq t<\bar{T}\}}$. At the age of retirement, which is 60 years, a lump sum of $a^{r}(\bar{T})=3$ is paid to all survivors. Finally, the contract contains a 30 year life annuity starting at age 60 with a rate of $a^{p}(t)=1$, which implies that $T=60$.

The parameters used for the mortality model are listed in Section 5.4, and the parameters for the financial market are listed in Table 4.

\begin{tabular}{cccc}
\hline \hline$r(0)$ & $\gamma^{r}$ & $\delta^{r}$ & $\sigma^{r}$ \\
\hline 0.03 & 0.011 & 0.2 & 0.01 \\
\hline \hline
\end{tabular}

Table 4: Parameters for the financial market.

\subsection{Trading of survivor swaps at initiation of the contract}

From Proposition 4.3 we get the discrete-time version of the Galtchouk-Kunita-Watanabe decomposition, and we see that the optimal number of survivor swaps, when we can trade at time 0 only, is given by

$$
\widehat{\vartheta}_{j}^{A}(0)=\frac{\mathrm{E}^{Q}\left[\int_{0}^{T} \vartheta_{j}^{A}(u) d L^{Z_{j}^{*}, Q}(u) \Delta L^{Z_{j}^{*}, Q}(T) \mid \mathcal{F}(0)\right]}{\mathrm{E}^{Q}\left[\left(\Delta L^{Z_{j}^{*}, Q}(T)\right)^{2} \mid \mathcal{F}(0)\right]} .
$$

Here, $\vartheta_{j}^{A}$ is determined from the continuous time Galtchouk-Kunita-Watanabe decomposition (3.3) of the intrinsic value process. Explicit expressions can be found in Dahl et al. (2008, Propositions 5.1 and 5.2) and are repeated here for completeness:

$$
\begin{aligned}
\vartheta_{1}^{A}(t) & =\frac{\nu^{V, Q}(t)+\rho_{1}^{V, Q}(t)\left(\kappa_{1,1}^{Q}(t)\right)^{-1} \chi_{1,1}^{Z, Q}(t)+\rho_{2}^{V, Q}(t)\left(\kappa_{1,2}^{Q}(t)\right)^{-1} \chi_{1,2}^{Z, Q}(t)}{\nu_{1}^{Z, Q}(t)+\rho_{1,1}^{Z, Q}(t)\left(\kappa_{1,1}^{Q}(t)\right)^{-1} \chi_{1,1}^{Z, Q}(t)+\rho_{1,2}^{Z, Q}(t)\left(\kappa_{1,2}^{Q}(t)\right)^{-1} \chi_{1,2}^{Z, Q}(t)}, \\
\vartheta_{2}^{A}(t) & =\frac{\rho_{1}^{V, Q}(t)\left(\kappa_{2,1}^{Q}(t)\right)^{-1} \chi_{2,1}^{Z, Q}(t)+\rho_{2}^{V, Q}(t)\left(\kappa_{2,2}^{Q}(t)\right)^{-1} \chi_{2,2}^{Z, Q}(t)}{\nu_{2}^{Z, Q}(t)+\rho_{2,1}^{Z, Q}(t)\left(\kappa_{2,1}^{Q}(t)\right)^{-1} \chi_{2,1}^{Z, Q}(t)+\rho_{2,2}^{Z, Q}(t)\left(\kappa_{2,2}^{Q}(t)\right)^{-1} \chi_{2,2}^{Z, Q}(t)},
\end{aligned}
$$

where for $\rho_{i, j}^{Z, Q}(t) \neq 0$,

$$
\kappa_{i, j}^{Q}(t)=\frac{\nu_{i}^{Z, Q}(t) \lambda_{i}^{Q}(x, t)}{\rho_{i, j}^{Z, Q}(t)} .
$$

and $\chi_{i, j}^{Z, Q}(t)=1_{\left\{\rho_{i, j}^{Z, Q}(t) \neq 0\right\}}$, for $i, j \in\{1,2\}$. For interpretation of these quantities, we refer to Dahl et al. (2008).

Furthermore, $\Delta L^{Z_{j}^{*}, Q}(T)=L^{Z_{j}^{*}, Q}(T)-L^{Z_{j}^{*}, Q}(0)$. The orthogonal parts in the decomposition (4.1) for $d Z_{j}^{*, Q}$ can be found in Lemma 5.2, and are given by

$$
L^{Z_{j}^{*}, Q}(T)=\int_{0}^{T} \nu_{j}^{Z, Q}(u) d M_{j}^{Q}(x, u)+\int_{0}^{T} \rho_{j}^{Z, Q}(u) d W^{\mu, Q}(u) .
$$


In order to calculate $\widehat{\vartheta}_{j}^{A}(0)$ in (6.1) we apply Monte Carlo simulation. Table 5 shows the results for different values of $n_{1}$ and $n_{2}$. The numbers in the table reflect the interaction

\begin{tabular}{rccc}
\hline \hline$n_{1}$ & $n_{2}$ & Portfolio (1) & Portfolio (2) \\
\hline 100 & 1,000 & 0.14 & 0.033 \\
100 & 10,000 & 0.14 & 0.0052 \\
1,000 & 10,000 & 0.39 & 0.051 \\
1,000 & 100,000 & 0.39 & 0.0055 \\
10,000 & 100,000 & 0.56 & 0.055 \\
\hline \hline
\end{tabular}

Table 5: Number of survivor swaps $\widehat{\vartheta}_{j}^{A}(0)$ in the market $\left(B, P, Z_{j}\right)$, where we can trade the survivor swap $Z_{j}$ at time 0 only (based on 10,000 simulations).

between systematic and unsystematic mortality risk in the insurance portfolio and the survivor swaps. First, we note that $\widehat{\vartheta}_{1}^{A}(0)$ is independent of $n_{2}$ as expected. In addition, we see that the optimal number of survivor swaps on portfolio 1 increases as a function of $n_{1}$. We explain this by the fact that the unsystematic risk becomes negligible, as the size of the portfolio increases. In order to compare the optimal number of swaps on portfolio 2 , the results should be multiplied by the ratio $n_{2} / n_{1}$. After this multiplication, we see that the quantity increases with $n_{2}$, which may again be explained by the fact that the systematic mortality risk is the main mortality risk for large portfolios.

The discrete and continuous time investment strategies in three scenarios are depicted in Figure 3 for comparison for the case where $n_{1}=10.000$ and $n_{2}=100.000$. The figure
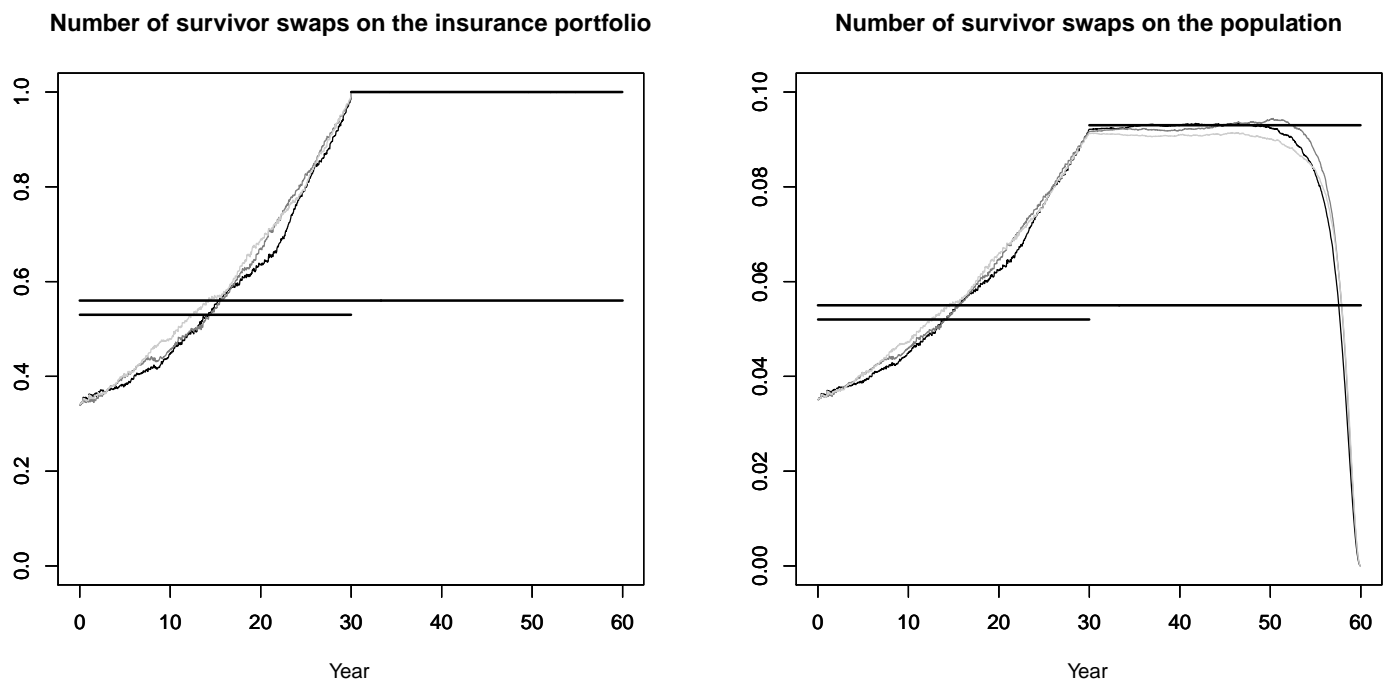

Figure 3: Number of survivor swaps in continuous and discrete time.

shows the optimal number of survivor swaps in the two situations where the insurance 
company has access to a survivor swap $Z_{1}$ on the company's own portfolio and a swap $Z_{2}$ on the population. For the situation where the number of swaps on the company's own portfolio (figure to the left) can be rebalanced dynamically (continuously), the optimal number of swaps increases from about 0.35 at time 0 to 1 at time 30 . After time 30, the optimal number remains constant and equal to 1 . The corresponding optimal constant strategy obtained from $(6.1)$ for the period $(0,60]$ is equal to 0.56 , which is included in Figure 3 for comparison. Finally, we have included a piecewise constant strategy, where the optimal number of swaps may be updated at time 30 using Proposition 4.3.

For the strategy based on the population swap (figure to the right), the optimal number of swaps at time 0 are around 0.035 and increases to around 0.093 at time 30. Shortly before time 60 , the strategy decreases to 0 . The corresponding constant strategy consists of buying 0.055 population swaps and holding these until the final term $T=60$. The

Survivor swaps on the insurance portfolio at time 0

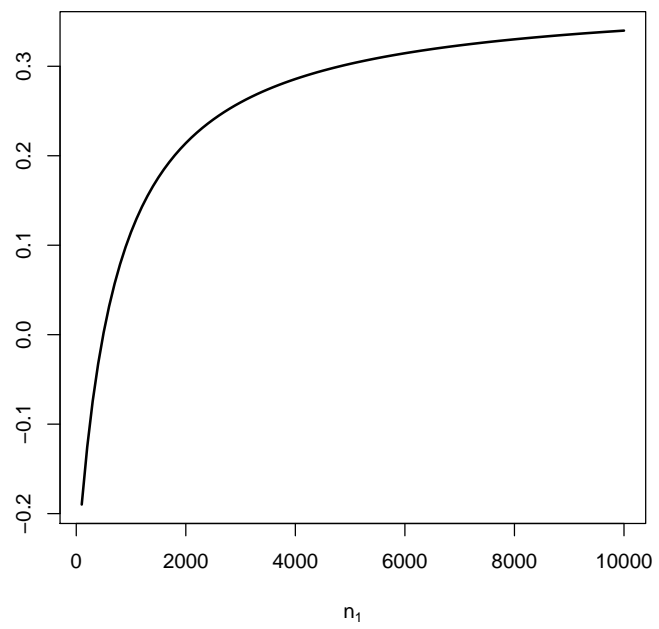

Survivor swaps on the population at time 0

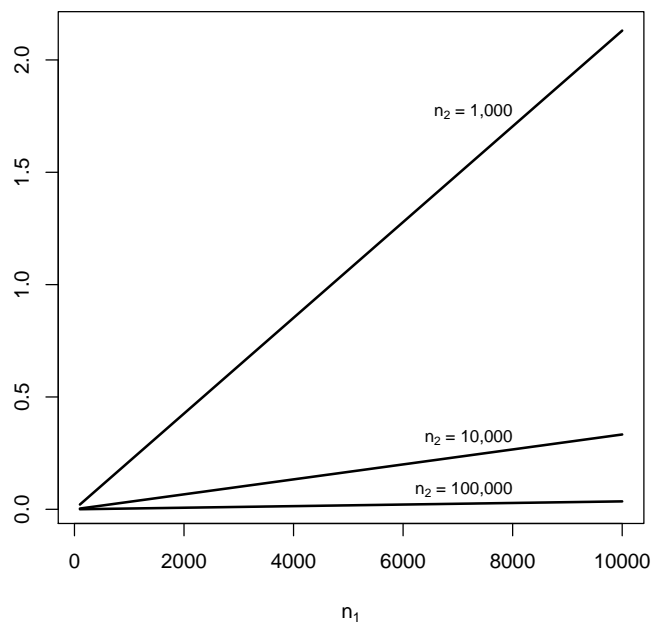

Figure 4: Number of survivor swaps at time 0 with a continuous strategy $\left(\vartheta_{j}^{A}(0)\right)$ as a function of the number of policy-holders $n_{1}$.

optimal strategies depend on the number of individuals in the two portfolios. We have illustrated this dependence in Figure 4. The left figure shows the optimal number of survivor swaps on portfolio 1 at time 0 , in the situation where the number of swaps may be updated continuously. The optimal number increases as a function of $n_{1}$ and converges to about 0.35 ; for small value of $n_{1}$, the optimal number at time 0 is negative. These differences may be explained by the relation between unsystematic and systematic mortality risk. The right figure highlights the fact that the optimal number of swaps on portfolio 2 essentially increases linearly with $n_{1}$ for fixed $n_{2}$.

\subsection{Trading of survivor swaps at initiation of the contract and retire- ment}

We apply Proposition 4.3 for calculating the number of swaps at time 0 and 30 . One could have chosen to calculate the number of swaps at time 30 by taking into account the development until time 30 in each case. This is not done here though due to computational performance issues. The numerical results can be found in Table 6 . We note that a 


\begin{tabular}{cccccc}
\hline \hline$n_{1}$ & $n_{2}$ & $\begin{array}{c}\text { Portfolio (1) } \\
t=0\end{array}$ & $\begin{array}{c}\text { Portfolio (1) } \\
t=30\end{array}$ & $\begin{array}{c}\text { Portfolio (2) } \\
t=0\end{array}$ & $\begin{array}{c}\text { Portfolio (2) } \\
t=30\end{array}$ \\
\hline 100 & 1,000 & 0.03 & 1 & 0.032 & 0.051 \\
100 & 10,000 & 0.03 & 1 & 0.0049 & 0.0086 \\
1,000 & 10,000 & 0.32 & 1 & 0.049 & 0.086 \\
1,000 & 100,000 & 0.32 & 1 & 0.0052 & 0.0093 \\
10,000 & 100,000 & 0.53 & 1 & 0.052 & 0.093 \\
\hline \hline
\end{tabular}

Table 6: Number of survivor swaps $\widehat{\vartheta}_{j}^{A}(t)$ in the market $\left(B, P, Z_{j}\right)$, where we can trade the survivor swap $Z_{j}$ at time 0 and 30 only (based on 10,000 simulations).

perfect hedge is obtained by holding exactly 1 survivor swap on the insurance portfolio from $t=30$. If we compare the optimal number of swaps for the interval $(0,30]$ with the optimal number for the interval $(0,60]$ in Table 5 , we see a relatively small difference for portfolio 2. For portfolio 1, the largest differences appear for small portfolio sizes. The discrete and continuous time investment strategies for the three scenarios are collected in Figure 3 for comparison. Here we see that the hedging strategies with rebalancing of the number of swaps at time 30 are much more similar to the optimal strategies with continuous rebalancing of the swaps.

\section{Comparison of the intrinsic risk at time 0}

In this section we compare the efficiency of different strategies. The comparison is carried out by calculating and comparing the intrinsic risks at time $0, R(0, \cdot)$ in different markets.

First we concentrate on the discrete-time case. In order to calculate intrinsic risk at time 0 in the discrete-time case, we keep $\widehat{\vartheta}_{j}^{A}(t)$ constant and equal to $\widehat{\vartheta}_{j}^{A}(0)$ in the time interval $[0, T]$. From the unhedgeable part in (4.13) we have that the intrinsic risk can be calculated as

$$
R^{\text {disc }}(0)=R^{\text {cont }}(0)+R^{\text {extra }}(0),
$$

where

$$
R^{\mathrm{cont}}(0)=\mathrm{E}^{Q}\left[\left(\int_{0}^{T} d L^{A}(u)\right)^{2}\right]
$$

is the intrinsic risk from continuous time, and

$$
R^{\text {extra }}(0)=\mathrm{E}^{Q}\left[\int_{0}^{T}\left(\vartheta^{A}(u)-\widehat{\vartheta}^{A}(0)\right)^{2} d\left\langle L^{Z_{j}^{*}, Q}\right\rangle(u)\right]
$$

is the extra term from (4.13) concerning the additional discrete-time part. When we carry out the numerical integration, we use that

$$
\begin{aligned}
d\left\langle L^{Z_{j}^{*}, Q}\right\rangle & =\left(\nu_{j}^{Z, Q}\right)^{2} d\left\langle M_{j}\right\rangle+\left(\rho_{j}^{Z, Q}\right)^{2} d\left\langle W^{\mu, Q}\right\rangle \\
& =\left(\left(\nu_{j}^{Z, Q}\right)^{2} \lambda_{j}+\left(\rho_{j, 1}^{Z, Q}\right)^{2}+\left(\rho_{j, 2}^{Z, Q}\right)^{2}\right) d t .
\end{aligned}
$$


The results can be found later in Table 7 along with the intrinsic risks from the continuous time cases.

We start out by writing down the intrinsic risk processes for the basic markets $(B)$ and $(B, P)$. First, we consider the market $(B)$, where we can invest only in the savings account $B$. Then from Lemma 5.1 and the theory of risk-minimization, we get the intrinsic risk process

$$
R\left(t, \varphi_{V}^{*}\right)=\mathrm{E}^{Q}\left[\int_{t}^{T}\left(\left(\nu^{V, Q}(\tau)\right)^{2} \lambda_{1}(x, \tau)+\left(\eta^{V, Q}(\tau)\right)^{2}+\sum_{j=1}^{2}\left(\rho_{j}^{V, Q}(\tau)\right)^{2}\right) d \tau \mid \mathcal{F}(t)\right] .
$$

The intrinsic risk process for the $(B, P)$-market containing the savings account and a zero coupon bond is taken from Dahl et al. (2008), and it is given by

$$
R\left(t, \varphi_{B}^{*}\right)=\mathrm{E}^{Q}\left[\int_{t}^{T}\left(\left(\nu^{V, Q}(\tau)\right)^{2} \lambda_{1}(x, \tau)+\sum_{j=1}^{2}\left(\rho_{j}^{V, Q}(\tau)\right)^{2}\right) d \tau \mid \mathcal{F}(t)\right] .
$$

Similarly, the intrinsic risk processes for the markets containing the survivor swaps are given by

$$
\begin{aligned}
R\left(t, \varphi_{1}^{*}\right)= & E^{Q}\left[\left(\int_{t}^{T}\left(\nu^{V, Q}(\tau)-\vartheta_{1}^{A}(\tau) \nu_{1}^{Z, Q}(\tau)\right)^{2} \lambda_{1}^{Q}(x, \tau)\right.\right. \\
& \left.\left.+\sum_{j=1}^{2}\left(\rho_{j}^{V, Q}(\tau)-\vartheta_{1}^{A}(\tau) \rho_{1, j}^{Z, Q}(\tau)\right)^{2}\right) d \tau \mid \mathcal{F}(t)\right]
\end{aligned}
$$

and

$$
\begin{aligned}
R\left(t, \varphi_{2}^{*}\right)= & E^{Q}\left[\left(\int_{t}^{T}\left(\nu^{V, Q}(\tau)\right)^{2} \lambda_{1}^{Q}(x, \tau)+\left(\vartheta_{2}^{A}(\tau) \nu_{2}^{Z, Q}(\tau)\right)^{2} \lambda_{2}^{Q}(x, \tau)\right.\right. \\
& \left.\left.+\sum_{j=1}^{2}\left(\rho_{j}^{V, Q}(\tau)-\vartheta_{2}^{A}(\tau) \rho_{2, j}^{Z, Q}(\tau)\right)^{2}\right) d \tau \mid \mathcal{F}(t)\right] .
\end{aligned}
$$

In Table 7 we have listed the intrinsic risks calculated at time 0 in the different markets. Note that $\varphi_{j}^{*}$ represents continuous time trading with survivor swap $j$, and $\varphi_{D_{j}^{i}}^{*}$ is discretetime trading, where $D_{j}^{1}$ indicates that the survivor swap can be traded at time 0 only, and $D_{j}^{2}$ indicates that the survivor swap can be traded both at time 0 and 30 .

First we note, that $\varphi_{V}^{*}, \varphi_{B}^{*}, \varphi_{1}^{*}, \varphi_{D_{1}^{1}}^{*}$ and $\varphi_{D_{1}^{2}}^{*}$ are independent of $n_{2}$. So the numbers in case 1 and 2 and case 3 and 4 are equal for the markets, which do not involve the swap on the population.

It is shown in Dahl et al. (2008) that extending the market to contain a zero coupon bond eliminates all the financial risk generated by $W^{r}$, and we see in Table 7 that this is by far the most significant risk factor. Furthermore, we observe that in the $(B, P)$-market, the intrinsic risk decreases when the portfolio gets larger, due to diversification of the unsystematic mortality risk.

When we introduce the different types of survivor swaps, we see that even more risk can be eliminated. First of all we note that in a continuous time trading setup, the survivor 


\begin{tabular}{rrrrrr}
\hline \hline$n_{1}$ & $n_{2}$ & $\frac{\sqrt{R\left(0, \varphi_{V}^{*}\right)}}{n_{1}}$ & $\frac{\sqrt{R\left(0, \varphi_{B}^{*}\right)}}{n_{1}}$ & $\frac{\sqrt{R\left(0, \varphi_{1}^{*}\right)}}{n_{1}}$ & $\frac{\sqrt{R\left(0, \varphi_{2}^{*}\right)}}{n_{1}}$ \\
\hline 100 & 1,000 & 0.634 & 0.111 & 0.048 & 0.103 \\
100 & 10,000 & 0.634 & 0.111 & 0.048 & 0.098 \\
1,000 & 10,000 & 0.627 & 0.062 & 0.032 & 0.037 \\
1,000 & 100,000 & 0.627 & 0.062 & 0.032 & 0.035 \\
10,000 & 100,000 & 0.626 & 0.055 & 0.013 & 0.020 \\
\hline \hline$n_{1}$ & $n_{2}$ & $\frac{\sqrt{R\left(0, \varphi_{D_{1}^{*}}^{*}\right)}}{n_{1}}$ & $\frac{\sqrt{R\left(0, \varphi_{D_{2}^{*}}^{*}\right)}}{n_{1}}$ & $\frac{\sqrt{R\left(0, \varphi_{D_{1}^{2}}^{*}\right)}}{n_{1}}$ & $\frac{\sqrt{R\left(0, \varphi_{D_{2}^{*}}^{*}\right)}}{n_{1}}$ \\
\hline 100 & 1,000 & 0.105 & 0.104 & 0.073 & 0.104 \\
100 & 10,000 & 0.105 & 0.100 & 0.073 & 0.100 \\
1,000 & 10,000 & 0.045 & 0.040 & 0.038 & 0.039 \\
1,000 & 100,000 & 0.045 & 0.039 & 0.038 & 0.037 \\
10,000 & 100,000 & 0.022 & 0.026 & 0.019 & 0.024 \\
\hline \hline
\end{tabular}

Table 7: Intrinsic risks calculated at time 0 , where $D_{j}^{1}$ and $D_{j}^{2}$ indicate that the survivor swap can be traded at time 0 only and at time 0 and 30, respectively (based on 5,000 simulations).

swap on the insurance portfolio is superior to the survivor swap based on the population. This is due to the fact that the swap on the insurance portfolio hedges the systematic mortality risk closer than the swap on the population, because it takes $W_{1}^{\mu}$ into account too; see Table 2. Beside that, it hedges against unsystematic mortality risk, whereas the swap on the population actually adds a new source of unsystematic mortality risk to the market.

Considering the discrete-time trading scenarios, we see that an interesting point appears. When we can trade the survivor swap on the insurance portfolio at time 0 only, we loose the ability to react to unsystematic mortality changes, and in that case we actually get a lower intrinsic risk by investing in the survivor swap on population, compared to investing in the swap on insurance portfolio, when the portfolio is small. In general, we see that when the portfolio gets smaller the unsystematic mortality risk gets more significant. Furthermore, we see that being able to trade at time 30 also reduces the intrinsic risk significantly for the swap on the portfolio, whereas the swap on the population is more or less unaffected.

\section{References}

Björk, T. (2004). Arbitrage Theory in Continuous Time, 2nd edn, Oxford University Press.

Cairns, A., Blake, D. and Dowd, K. (2008). Modelling and management of mortality risk: a review, Scandinavian Actuarial Journal, 79-113.

Dahl, M. (2004). Stochastic Mortality in Life Insurance: Market Reserves and Mortality-Linked Insurance Contracts, Insurance: Mathematics and Economics 35, 113-136. 
Dahl, M., Melchior, M. and Møller, T. (2008). On Systematic Mortality Risk and RiskMinimization with Survivor Swaps, Scandinavian Actuarial journal 2008, 114-146.

Dahl, M. and Møller, T. (2006). Valuation and Hedging of Life Insurance Liabilities with Systematic Mortality Risk, Insurance: Mathematics and Economics 39, 193-217.

Dowd, K., Blake, D., Cairns, A. and Dawson, P. (2006). Survivor Swaps, Journal of Risk and Insurance 73, 1-17.

Föllmer, H. and Sondermann, D. (1986). Hedging of Non-Redundant Contingent Claims, in W. Hildenbrand and A. Mas-Colell (eds), Contributions to Mathematical Economics, NorthHolland, 205-223.

Møller, T. (2001). Risk-Minimizing Hedging Strategies for Insurance Payment Processes, Finance and Stochastics 5(4), 419-446.

Schweizer, M. (1994). Risk-minimizing Hedging Strategies under Restricted Information, Mathematical Finance 4, 327-342.

Schweizer, M. (2001). A Guided Tour through Quadratic Hedging Approaches, in E. Jouini, J. Cvitanić and M. Musiela (eds), Option Pricing, Interest Rates and Risk Management, Cambridge University Press, 538-574. 